\title{
$\angle$ Research Square \\ Uric acid levels are critical for non-alcoholic fatty liver disease
}

\section{Guanqun Chao}

Zhejiang University School of Medicine Sir Run Run Shaw Hospital

Yue Zhu

Lizheng Fang (D 3197056@zju.edu.cn )

\section{Research}

Keywords: non-alcoholic fatty liver disease, uric acid, risk factor

Posted Date: February 12th, 2020

DOl: https://doi.org/10.21203/rs.2.23270/v1

License: (a) (i) This work is licensed under a Creative Commons Attribution 4.0 International License. Read Full License 


\section{Abstract}

Background: To clarify the risk factors associated with NAFLD and further clarify the correlation between uric acid level and NAFLD by analyzing the correlation between NAFLD and different metabolic factors.

Methods: Datas were obtained from subjects who underwent health examination in the Health promotion centre of Sir Run Run Shaw hospital of Zhejiang University from January 2016 to December 2017.The diagnosis of NAFLD was according to the clinical diagnosis of the Guidelines.Statistical analyses were performed using R software.

Results: 79492 subjects were analyzed. 56680(71.3\%) participants did not have NAFLD, 22812(28.7\%) participants had NAFLD. Male, age, BMI, high blood pressure, central obesity, high glycosylated hemoglobin, high serum uric acid, high triglyceride, high total cholesterol, high low density lipoprotein cholesterol (LDL-C), abnormal liver function were risk factors of NAFLD, however, low high-density lipoprotein cholesterol (HDL-C) level was another risk factor of NAFLD.OR value suggested serum uric acid was a robust risk factor for NAFLD in all subgroups.In male group, AUC was 0.656 (95\% Cl: 0.651-0.661), the optimal diagnostic threshold was $395.5 \mathrm{~mol} / \mathrm{L}$, the sensitivity was $61.9 \%$, the specificity was $61.1 \%$, and the yoden index was 0.23 . In female group, AUC was $0.716(95 \% \mathrm{Cl}$ : $0.708-0.724)$, the optimal diagnostic threshold was $294.5 \mathrm{~mol} / \mathrm{L}$, sensitivity was $67.7 \%$, specificity was $64.5 \%$, and the Jordan index was 0.32 .

Conclusions: Our study suggested that there was a close correlation between serum uric acid level and NAFLD.Uric acid levels was a key risk factor for NAFLD.The diagnosis of fatty liver in patients can be preliminarily determined by detecting uric acid level.

\section{Contributions to the literature》}

1. The purpose of this study was to clarify the risk factors associated with NAFLD and further clarify the correlation between uric acid level and NAFLD by analyzing the correlation between NAFLD and different metabolic factors in the physical examination population.

2. There was a close correlation between serum uric acid level and NAFLD.Uric acid levels was a key risk factor for NAFLD.

3. The diagnosis of fatty liver in patients can be preliminarily determined by detecting uric acid level.

\section{Background}

Non-alcoholic fatty liver disease (NAFLD) is a kind of liver disease without viral hepatitis, drug-induced liver injury, excessive alcohol consumption, hereditary disorders and so on, as a result of its high prevalence it becomes an important public health issue ${ }^{1}$. The prevalence varies between $15 \%$ and $30 \% 20$ in China and $30 \%$ in western countries $^{2}$. The prevalence among obese individuals is $75 \%^{3}$. NAFLD is associated with impaired glucose tolerance, imbalance in lipid homeostasis, dyslipidemia, cardiovascular disease, abnormalities in mitochondrial metabolism, and so on ${ }^{4}$. The progression of NAFLD will result in hepatic fibrosis, cirrhosis, liver failure, even hepatocellular carcinoma ${ }^{5}$ \and NAFLD has affected about $25 \%$ of the general population worldwide to be recognized as the most common cause of chronic liver disease ${ }^{6}$.NAFLD is also considered a leading cause of liver-related morbidity and mortality globally? 
During the last decade, the researchers have made remarkable advances in the understanding of the pathophysiology of NAFLD ${ }^{8}$. Once hepatic lipid accumulation overcomes the combination of intra-hepatic lipid oxidation and lipid export, NAFLD will progress ${ }^{9}$. NAFLD was found to be associated with multiple systemic diseases. NAFLD has proven to strongly correlate not only with metabolic syndrome, but also with type 2 diabetes,cardiovascular disease,chronic kidney disease and cardiovascular disease ${ }^{10-11}$. Not only that, NAFLD was found to be associated with increased incidence of colorectal adenomas ${ }^{12}$. As we know, obesity is considered to be an important risk factor for NAFLD. However, NAFLD can also occur in non-obese subjects. The gold standard for the diagnosis of NAFLD is biopsy, but biopsies are invasive and not all patients are willing to make a definitive diagnosis.As NAFLD is associated with many diseases and a risk factor for a variety of diseases, understanding the correlation between NAFLD and various factors is more conducive to understanding the risk of NAFLD. The research has reported positive associations between elevated SUA levels and lean-NAFLD risk in adults ${ }^{13}$. The purpose of this study was to clarify the risk factors associated with NAFLD and further clarify the correlation between uric acid level and NAFLD by analyzing the correlation between NAFLD and different metabolic factors in the physical examination population.

\section{Method}

\subsection{Data and methods}

Datas were obtained from subjects who underwent health examination in the Health promotion centre of Sir Run Run Shaw hospital of Zhejiang University from January 2016 to December 2017(Figure 1). Exclusion analysis criteria:1) Age<18y or age>80y; 2) Repeat physical examination population;3) Alcohol consumption>140 g/week for men and $70 \mathrm{~g} /$ week for women; 4) Subjects using hypertension, diabetes, hyperlipidemia and other metabolic diseases drug treatment; 5) Subjects with a history of hepatitis or autoimmune hepatitis; 6) Lack of information.. The study was approved by Ethics and Human Subject Committee of Zhejiang University.Liver ultrasonography was performed by experienced technicians. The diagnosis of NAFLD was according to the clinical diagnosis of the Guidelines[14].

\subsection{Statistical analyses}

Statistical analyses were performed using R software (version 3.5.1; The R Foundation for Statistical Computing).Continuous variables with normal distributions were expressed as the mean \pm standard deviation $(\mathrm{X} \pm \mathrm{SD})$, and the classification variables were expressed as percentages. T-test was used for mean comparison between the two groups, and chi-square test was used for counting data comparison. Multivariate Logistic regression was used to analyze the ratio ratio (OR value) of age, gender, blood pressure, blood lipid and other categorical variables. The diagnostic performance of the SUA was assessed using the receiver operating characteristic(ROC) curve. All statistical analyses were two-sided. When p value was less than 0.05 , statistical significance was indicated.

\section{Results}

\subsection{Demographic and laboratory variables of the studied subjects}

In total, 79492 subjects were analyzed in this study. A total of 56680(71.3\%) participants did not have NAFLD, 22812(28.7\%) participants had NAFLD.According to the results of ultrasound, the subjects were divided into NAFLD 
group and control group. BMl,Age,systolic pressure(SBP), diastolic pressure(DBP),waist circumference(WC),Uric Acid(UA), total cholesterol (TC),triglyceride(TG),Hba1c,ALT,AST,GGT,LDLC,creatinine(CR),blood urea nitrogen(BUN) were significantly higher in subjects with NAFLD than in controls.Further more, HDL-C was significantly lower in subjects with NAFLD than in controls.(Table 1)

\subsection{Logistic regression analysis of risk factors}

Existing variables were divided into groups: (1) Male and female groups according to gender;(2) 5 age groups: 1830 group,30-40 group,40-50 group,50-60 group,60-80 group;(3) Blood pressure groups: hypertension group (SBP $\geq 140 \mathrm{mmHg}$ or DBP $\geq 90 \mathrm{mmHg}$ ) and normal blood pressure group;(4) Waist circumference groups:central obesity (male waist $>90 \mathrm{~cm}$ or female waist $>80 \mathrm{~cm}$ ) and the normal group; (5) According to the levels of ALT and AST, the subjects were divided into abnormal liver function (ALT $>40 \mathrm{IU} / \mathrm{L}$ or $A S T>40 \mathrm{IU} / \mathrm{L}$ ) group and the normal group.(6) According to the blood lipid level, divided into high TC group (TC $5.2 \mathrm{mmol} / \mathrm{L}$ ), high TG group (TG 1.7 $\mathrm{mmol} / \mathrm{L}$ ), low HDL group (hdl-c $<1.00 \mathrm{mg} / \mathrm{dL}$ ), high LDL group (Idl-c $3.4 \mathrm{mmol} / \mathrm{L}$ ). (7) Serum uric acid groups by arbitrary quartile (Q1 to Q4) : $<275 \mathrm{mg} / \mathrm{dL}, 275 \mathrm{mg} / \mathrm{dL}-336 \mathrm{mg} / \mathrm{dL}, 336 \mathrm{mg} / \mathrm{dL}-402 \mathrm{mg} / \mathrm{dL},>402 \mathrm{mg} / \mathrm{dL}$.

In order to better determine the risk factors of NAFLD, we conducted Logistics regression analysis.From single factor logistic regression models, male, age, BMI, high blood pressure, central obesity, high glycosylated hemoglobin, high serum uric acid, high triglyceride, high total cholesterol, high low density lipoprotein cholesterol (LDL-C), abnormal liver function were risk factors of NAFLD, however, low high-density lipoprotein cholesterol (HDLC) level was another risk factor of NAFLD.Among them, the ratio of NAFLD in the high serum uric acid level group was higher than that in the lowest serum uric acid level group, and the OR of the Q2-Q4 group were 6.039 (95\%ci 5.393-6.788), 25.830 (95\%ci 22.950-29.172), and 59.308 (95\%ci 38.574-94.116). Factors with $\mathrm{P}<0.05$ in univariate analysis (gender, age, BMl, hypertension, central obesity, diabetes, high serum uric acid level, high triglyceride, high total cholesterol, high low density lipoprotein, low high density lipoprotein, abnormal liver function) were included in the logistic multivariate regression model for analysis.In the multi-factor Logistics regression analysis, the conclusions were similar. The OR of group Q2-Q4 were 2.616 (95\%ci 2.300-2.986), 4.444 (95\%ci 3.866-5.123) and 7.879 (95\%ci 4.722-13.479)(Table 2).

\subsection{Fractional analysis}

According to different gender, age, BMI, hypertension, central obesity, diabetes, lipid profile and liver function, the ratio of NAFLD among subjects with high uric acid level (male $>420 \mathrm{mg} / \mathrm{dL}$, female $>360 \mathrm{mg} / \mathrm{dL}$ ) and those with low uric acid level were calculated.In different group(different gender, age, BMI, blood pressure levels, central obesity, triglycerides, high-density lipoprotein cholesterol, low density lipoprotein cholesterol, liver function), the OR value of NAFLD in subjects with high uric acid level was greater than 1, which means serum uric acid was a robust risk factor for NAFLD in all subgroups(Figure 2).

\subsection{ROC curve analysis}

The diagnostic value of serum uric acid in NAFLD was further analyzed by ROC curve.Gender, age, BMI, hypertension, central obesity, diabetes, serum uric acid levels, high triglycerides, high total cholesterol, high LDL-C, low HDL-C, and liver dysfunction were all independent risk factors for NAFLD. The AUC of serum uric acid ROC curve was 0.7488 (95\% Cl: $0.7451-0.7524$ ), the optimal diagnostic threshold was $342.5 \mathrm{~mol} / \mathrm{L}$, the sensitivity was $63.5 \%$, the specificity was $73.9 \%$, and the yoden index was 0.37 , which is higher than the predictors such as HbA1c, TC, HDL, LDL and SBP(Table 3). 
We further analyzed the diagnostic value of serum uric acid for NAFLD in different gender group.In male group, NAFLD was diagnosed according to serum uric acid level, ROC curve and diagnostic boundary value, AUC was 0.656 (95\% Cl: $0.651-0.661)$, the optimal diagnostic threshold was $395.5 \mathrm{~mol} / \mathrm{L}$, the sensitivity was $61.9 \%$, the specificity was $61.1 \%$, and the yoden index was 0.23 (Figure 3 ). In female group, AUC was 0.716 (95\%Cl: 0.708$0.724)$, the optimal diagnostic threshold was $294.5 \mathrm{~mol} / \mathrm{L}$, sensitivity was $67.7 \%$, specificity was $64.5 \%$, and the Jordan index was 0.32(Figure 4).

\section{Discussion}

In our study, we observed a positive relationship among uric acid, other related indicators and NAFLD. Our findings showed the prevalence of NAFLD was $28.7 \%$, which is higher than the prevalence $(14.7 \%)$ in another study ${ }^{15}$. A recent study ${ }^{16}$ showed the same prevalence of NAFLD (27.6\%), howere, the subjects were nondiabetic Chinese men living in coastal areas. The possible reason of different prevalence might be due to the different characteristics of the selected candidates and different research eras.NAFLD has been reported to be prevalent in $20 \%-30 \%$ of the general population that is same to our study; and the prevalence reaches $70 \%-90 \%$ in obesity or diabetes patients ${ }^{17}$.It shows that obesity and blood sugar could be the risk factors for NAFLD that the result is consistent with our study. Our study showed that the higher of BMI,Age,systolic pressure(SBP), diastolic pressure(DBP),waist circumference(WC),Uric Acid(UA), total cholesterol (TC),triglyceride(TG),Hba1c,ALT,AST,GGT,LDLC,creatinine(CR),blood urea nitrogen(BUN) or the lower of HDL-C would lead to the higher prevalence of NAFLD. As a result, obesity, hypertension, hyperglycemia, hyperlipidemia, liver dysfunction and hyperuricemia are positively correlated with the incidence of NAFLD.

In order to better determine the risk factors of NAFLD, we conducted Logistics regression analysis. Our study showed that male, age, high blood pressure, central obesity, high glycosylated hemoglobin, high serum uric acid, high triglyceride, high total cholesterol, high low density lipoprotein cholesterol (LDL-C), low high-density lipoprotein cholesterol (HDL-C) , abnormal liver function were risk factors of NAFLD. And Crucially, the incidence of NAFLD is positively correlated with increased uric acid level. However, age $<30$ or $\mathrm{UA}<275 \mathrm{mg} / \mathrm{dL}$ is neither the risk factor nor the protective factor for NAFLD, and is not associated with the onset of NAFLD. obesity and elevated UA have been reported to synergistically interact to increase the risk of NAFLD ${ }^{18}$ wihch is consistent with our study. 8925 subjects in East China's Zhejiang province were sutdied to report that hyperuricemia was related to NAFLD and SUA levels were independently and positively associated with the risk for incident NAFLD ${ }^{19}$. Another study ${ }^{16}$ reported that Increased serum uric acid levels might be involved in the development of NAFLD in men presenting with metabolic syndrome and/or insulin resistance. All results suggest that high uric acid is associated with the incidence of NAFLD, but no association has been established between low uric acid and low incidence of NAFLD. Our study found that when uric acid $\geq 275 \mathrm{mg} / \mathrm{dL}$, the incidence of NAFLD increased significantly, while uric acid $<275 \mathrm{mg} / \mathrm{dL}$ did not reduce the occurrence of NAFLD. Therefore, at low uric acid levels, the reduction in the incidence of fatty liver cannot be reflected by lowering uric acid levels.

More and more studies have confirmed that elevated uric acid level is related to the occurrence of NAFLD, and it can be related through multiple pathways. Elevated UA level was reported to develope of insulin resistance (IR) by reducing endothelial nitric oxide(NO) bioavailability ${ }^{20}$. On the other hand, uric acid can cause liver steatosis through fructose metabolism ${ }^{21}$. Oxidative stress was reported to play a key role in uric acid-induced steatosis ${ }^{22}$.As a result of defects in arginine-dependent NO synthesis, gene knockout of Slc7a3a can cause hepatic steatosis ${ }^{23}$.In 
summary, uric acid can regulate lipid production, promote metabolic disorders and cause NAFLD through multiple pathways.

From our study, we found that uric acid was the most important risk factor for NAFLD in different subgroups. There is growing evidence that SUA levels are associated with NAFLD and even severe liver damage ${ }^{24}$ that are consistent with our study. At present, more and more researchers hope to look for non-invasive biological serological indexes to follow up the changes of NAFLD.Our study suggested that UA level could be used as the severitive marker of NAFLD. Atelet count ${ }^{25}$, hyaluronic acid levels ${ }^{26}$, neutrophil-to-lymphocyte ratio ${ }^{27}$ were reported to be connected with the presence of NAFLD. But other studies have found no correlation among them ${ }^{28,29}$.We used ROC curve to analyze the diagnostic value of serum indicators for NAFLD.AUC of UA level was higher than other serological indicators which meant UA level t could be considered as a noninvasive way to follow up NAFLD.We also used ROC curve to analyze the diagnostic significance of UA levels in male and female patients with NAFLD. The optimal diagnostic threshold of UA level is $395.5 \mu \mathrm{mol} / \mathrm{L}$ in male NAFLD patients, and the optimal diagnostic threshold of UA level is $294.5 \mu \mathrm{mol} / \mathrm{L}$ in female NAFLD patients. From our study, we considered the optimal diagnostic threshold can be used as the diagnostic standard value diagnose NAFLD.

There are some limitations in our study. First, The number of subjects included in the sample was not large enough, and all of them are in the southeast of China. Second, The study was unable to identify the diets of the study subjects. Third, there were some differences in the diagnosis of fatty liver by B-ultrasound.Therefore, further multicenter studies may be helpful.

\section{Conclusion}

In conclusion, our study suggested that there was a close correlation between serum uric acid level and NAFLD.Uric acid levels was a key risk factor for NAFLD. The diagnosis of fatty liver in patients can be preliminarily determined by detecting uric acid level.

\section{Abbreviations}

Non-alcoholic fatty liver disease (NAFLD);systolic pressure(SBP); diastolic pressure(DBP);waist circumference(WC),Uric Acid(UA);total cholesterol (TC);triglyceride(TG);creatinine(CR),blood urea nitrogen(BUN)

\section{Declarations}

\section{Compliance with Ethical Standards:}

Ethics approval and consent to participate区N/A凶This is a retrospective analysis.

Consent for publication $₫ \mathrm{~N} / \mathrm{A}$

Availability of data and materia/\$The data can searched by contacting the authors.

Competing interests: None

Funding $₫$ This research was supported by funding from Zhejiang Provincial Natural Science Foundation of China under Grant No.LY18H030001囚the Medicine and Health Science and Technology Plan Projects in Zhejiang province(2017KY413), Traditional Chinese Medicine Science and Technology Plan of Zhejiang 
Province(2017ZA089, 2016ZB071, 2015ZZ012, 2014ZA030); National Natural Science Foundation of China (81573760);Medical Health Platform Plan Projects of Zhejiang Province (2015RCA020); Zhejiang Provincial Natural Science Foundation of China (LY16H030010).

Authors' contributions: Guanqun Chao wrote the analysis, Yue Zhu did the analysis, Lizheng Fang guided.

Acknowledgements: None

\section{References}

1. Chen Z, Liu F, Zheng N, Guo M, Bao L, Zhan Y, Zhang M, Zhao Y, Guo W, Ding G.Wuzhi capsule (Schisandra Sphenanthera extract) attenuates liver steatosis and inflammationduring nonalcoholic fatty liver disease development.Biomed Pharmacother.2018;110:285-293.

2. Loomba R, Sanyal AJ.The global NAFLD epidemic.Nat Rev Gastroenterol 2013;10(11):686-90.

3. Review Team, LaBrecque DR, Abbas Z, Anania F, Ferenci P, Khan AG, Goh KL, Hamid SS, Isakov V, Lizarzabal M, Peñaranda MM, Ramos JF, Sarin S, Stimac D, Thomson AB, Umar M, Krabshuis J, LeMair A; World Gastroenterology

Organisation.World Gastroenterology Organisation global guidelines: Nonalcoholic fatty liver disease and nonalcoholic steatohepatitis.J Clin Gastroenterol.2014;48(6):467-73.

4. Breher-Esch S, Sahini N, Trincone A, Wallstab C, Borlak J.Genomics of lipid-laden human hepatocyte cultures enables drug target screening for the treatment of non-alcoholic fatty liver disease.BMC Med 2018;11(1):111.

5. Yilmaz B, Sahin K, Bilen H, Bahcecioglu IH, Bilir B, Ashraf S, Halazun KJ, Kucuk O.Carotenoids and nonalcoholic fatty liver disease.Hepatobiliary Surg 2015;4(3):161-71.

6. Younossi ZM, Koenig AB, Abdelatif D, Fazel Y, Henry L, Wymer M.Global epidemiology of nonalcoholic fatty liver disease-Meta-analytic assessment of prevalence, incidence, and outcomes.2016;64(1):73-84.

7. Lonardo A, Nascimbeni F, Maurantonio M, Marrazzo A, Rinaldi L, Adinolfi LE.Nonalcoholic fatty liver disease: Evolving paradigms. World J Gastroenterol.2017;23(36):6571-6592.

8. Arab JP, Arrese M, Trauner M.Recent Insights into the Pathogenesis of Nonalcoholic Fatty Liver Disease.Annu Rev Pathol.2018;13:321-350.

9. Fotbolcu H, Zorlu E.Nonalcoholic fatty liver disease as a multi-systemic disease.World J Gastroenterol.2016;22(16):4079-90.

10. Musso G, Gambino R, Cassader M, Pagano G.Meta-analysis: natural history of nonalcoholic fatty liver disease (NAFLD) and diagnosticaccuracy of noninvasive tests for liver disease severity.Ann Med.2011;43(8):617-49.

11. Mantovani A, Zaza G, Byrne CD, Lonardo A, Zoppini G, Bonora E, Targher G.Nonalcoholic fatty liver disease increases risk of incident chronic kidney disease: A systematic review and meta-analysis.2018;79:64-76.

12. Wong VW, Wong GL, Tsang SW, Fan T, Chu WC, Woo J, Chan AW, Choi PC, Chim AM, Lau JY, Chan FK, Sung $\mathrm{JJ}$, Chan HL.High prevalence of colorectal neoplasm in patients with nonalcoholic steatohepatitis.2011;60(6):829-36.

13. Zheng X, Gong L, Luo R, Chen H, Peng B, Ren W, Wang Y.Serum uric acid and nonalcoholic fatty liver disease in non-obesity Chinese adults.Lipids Health Dis.2017;16(1):202. 
14. Chalasani N, Younossi Z, Lavine JE, Charlton M, Cusi K, Rinella M, Harrison SA, Brunt EM, Sanyal AJ.The diagnosis and management of nonalcoholic fatty liver disease: Practice guidance from the American Association for the Study of Liver Diseases.2018;67(1):328-357.

15. Li Y, Xu C, Yu C, Xu L, Miao M.Association of serum uric acid level with non-alcoholic fatty liver disease: a cross-sectional study.J Hepatol.2009;50(5):1029-34.

16. Xie Y, Wang M, Zhang Y, Zhang S, Tan A, Gao Y, Liang Z, Shi D, Huang Z, Zhang H, Yang X, Lu Z, Wu C, Liao M, Sun Y, Qin X, Hu Y, Li L, Peng T, Li Z, Yang X, Mo Z.Serum uric acid and nonalcoholic fatty liver disease in non-diabetic Chinese men.PLoS One.2013;8(7):e67152.

17. Vernon G, Baranova A, Younossi ZM.Systematic review: the epidemiology and natural history of non-alcoholic fatty liver disease and non-alcoholic steatohepatitis in adults.Aliment Pharmacol Ther.2011;34(3):274-85.

18. Zhang S, Du T, Li M, Lu H, Lin X, Yu X.Combined effect of obesity and uric acid on nonalcoholic fatty liver disease and hypertriglyceridemia.Medicine (Baltimore).2017;96(12):e6381.

19. Xu C, Yu C, Xu L, Miao M, Li Y.High serum uric acid increases the risk for nonalcoholic Fatty liver disease: a prospective observational study.PLoS One.2010;5(7):e11578.

20. Li C, Hsieh MC, Chang SJ.Metabolic syndrome, diabetes, and hyperuricemia.Curr Opin Rheumatol.2013;25(2):210-6.

21. Choi YJ, Shin HS, Choi HS, Park JW, Jo I, Oh ES, Lee KY, Lee BH, Johnson RJ, Kang DH.Uric acid induces fat accumulation via generation of endoplasmic reticulum stress and SREBP1cactivation in hepatocytes.Lab Invest.2014;94(10):1114-25.

22. Wan X, Xu C, Lin Y, Lu C, Li D, Sang J, He H, Liu X, Li Y, Yu C.Uric acid regulates hepatic steatosis and insulin resistance through the NLRP3 inflammasomedependent mechanism.J 2016;64(4):925-32.

23. Yoneda M, Fujii H, Sumida Y, Hyogo H, Itoh Y, Ono M, Eguchi Y, Suzuki Y, Aoki N, Kanemasa K, Imajo K, Chayama K, Saibara T, Kawada N, Fujimoto K, Kohgo Y, Yoshikawa T, Okanoue T; Japan Study Group of Nonalcoholic Fatty Liver Disease.Platelet count for predicting fibrosis in nonalcoholic fatty liver disease.J Gastroenterol.2011;46(11):1300-6.

24. Ryu S, Chang Y, Kim SG, Cho J, Guallar

E.Serum uric acid levels predict incident nonalcoholic fatty liver disease in healthy Korean men.2011;60(6):8606.

25. Yoneda M, Fujii H, Sumida Y, Hyogo H, Itoh Y, Ono M, Eguchi Y, Suzuki Y, Aoki N, Kanemasa K, Imajo K, Chayama K, Saibara T, Kawada N, Fujimoto K, Kohgo Y, Yoshikawa T, Okanoue T; Japan Study Group of Nonalcoholic Fatty Liver Disease.Platelet count for predicting fibrosis in nonalcoholic fatty liver disease.J Gastroenterol.2011;46(11):1300-6.

26. Kaneda H, Hashimoto E, Yatsuji S, Tokushige K, Shiratori K.Hyaluronic acid levels can predict severe fibrosis and platelet counts can predict cirrhosis in patients with nonalcoholic fatty liver disease.J Gastroenterol Hepatol.2006;21(9):1459-65.

27. Alkhouri N, Morris-Stiff G, Campbell C, Lopez R, Tamimi TA, Yerian L, Zein NN, Feldstein AE.Neutrophil to lymphocyte ratio: a new marker for predicting steatohepatitis and fibrosis in patients with nonalcoholic fatty liver disease.Liver 2012;32(2):297-302. 
28. Garjani A, Safaeiyan A, Khoshbaten M.Association between platelet count as a noninvasive marker and ultrasonographic grading in patients with nonalcoholic Fatty liver disease. Hepat Mon.2015;15(1):e24449.

29. Kara M, Dogru T, Genc H, Sertoglu E, Celebi G, Gurel H, Kayadibi H, Cicek AF, Ercin CN, Sonmez A.Neutrophil-tolymphocyte ratio is not a predictor of liver histology in patients with nonalcoholic fatty liver disease.Eur $\mathrm{J}$ Gastroenterol Hepatol.2015;27(10):1144-8.

\section{Tables}

Table 1 Demographic and laboratory variables of the studied subjects

\begin{tabular}{|c|c|c|c|c|}
\hline & $\begin{array}{c}\text { Control } \\
(\mathrm{n}=56680)\end{array}$ & $\begin{array}{c}\text { NAFLD } \\
(\mathrm{n}=22812)\end{array}$ & $\begin{array}{c}\text { Overall } \\
(\mathrm{n}=79492)\end{array}$ & $\begin{array}{c}\mathrm{P}- \\
\text { value }\end{array}$ \\
\hline Gender, \% & $44.4 \%$ & $77.8 \%$ & $42897(54.0 \%)$ & $<0.001$ \\
\hline years & $42.1 \pm 11.2$ & $45.2 \pm 10.4^{* * *}$ & $43.0 \pm 11.1$ & $<0.001$ \\
\hline $\mathrm{kg} / \mathrm{m}^{2}$ & $22.6 \pm 2.91$ & $25.8 \pm 3.13^{* * *}$ & $23.5 \pm 3.31$ & $<0.001$ \\
\hline \multicolumn{5}{|l|}{ d pressure, $\mathrm{mmHg}$} \\
\hline ystolic blood pressure & $117 \pm 14.9$ & $127 \pm 14.8^{* * *}$ & $120 \pm 15.5$ & $<0.001$ \\
\hline iastolic blood pressure & $70.8 \pm 10.3$ & $78.0 \pm 10.8^{* * *}$ & $72.9 \pm 11.0$ & $<0.001$ \\
\hline it circumference, $\mathrm{cm}$ & $78.0 \pm 8.89$ & $90.5 \pm 8.04^{* * *}$ & $81.6 \pm 10.3$ & $<0.001$ \\
\hline $1 c, \%$ & $5.23 \pm 0.482$ & $5.53 \pm 0.788^{* * *}$ & $5.32 \pm 0.603$ & $<0.001$ \\
\hline m Uric acid, mg/dL & $321 \pm 81.6$ & $399 \pm 86.4^{* * *}$ & $343 \pm 90.2$ & $<0.001$ \\
\hline 1 cholesterol, & $4.74 \pm 0.903$ & $5.12 \pm 0.998^{* * *}$ & $4.85 \pm 0.947$ & $<0.001$ \\
\hline lycerides, mg/dL & $1.27 \pm 0.913$ & $2.37 \pm 1.93^{* * *}$ & $1.58 \pm 1.38$ & $<0.001$ \\
\hline density lipoprotein & $2.68 \pm 0.731$ & $2.95 \pm 0.799^{* * *}$ & $2.76 \pm 0.761$ & $<0.001$ \\
\hline I density lipoprotein & $1.30 \pm 0.313$ & $1.06 \pm 0.241^{* * *}$ & $1.23 \pm 0.312$ & $<0.001$ \\
\hline ine aminotransferase, IU/L & $19.9 \pm 20.4$ & $36.9 \pm 29.2^{* * *}$ & $24.8 \pm 24.5$ & $<0.001$ \\
\hline irtate aminotransferase, IU/L & $20.4 \pm 11.7$ & $25.7 \pm 14.0^{* * *}$ & $21.9 \pm 12.6$ & $<0.001$ \\
\hline $\begin{array}{l}\text { ma Glutamyl } \mathrm{aIU} / \mathrm{L} \\
\text { speptidase, IU/L }\end{array}$ & $25.1 \pm 32.1$ & $49.8 \pm 51.1 * * *$ & $32.2 \pm 40.1$ & $<0.001$ \\
\hline itinine, $\mu \mathrm{mol} / \mathrm{L}$ & $67.5 \pm 15.6$ & $74.0 \pm 13.8^{* * *}$ & $69.3 \pm 15.4$ & $<0.001$ \\
\hline $\mathrm{d}$ urea nitrogen, $\mathrm{mmol} / \mathrm{L}$ & $4.60 \pm 1.17$ & $4.85 \pm 1.11^{* * *}$ & $4.67 \pm 1.16$ & $<0.001$ \\
\hline
\end{tabular}

Abbreviations: ALT alanine aminotransferase, AST aspartate aminotransferase, BMI body mass index, DBP diastolic blood pressure, HDL-c high density lipoprotein-cholesterol, LDL-c low-density lipoprotein-cholesterol, SBP systolic blood pressure, UA serum uric acid, TC total cholesterol, TG triacylglycerol ***means $\mathrm{P}<0.001$ compared with control group

Table 2 Logistics regression analysis for the risk factors of NAFLD 


\begin{tabular}{|c|c|c|c|c|c|c|}
\hline & \multicolumn{3}{|c|}{ Crude } & \multicolumn{3}{|c|}{ Adjusted* } \\
\hline & OR & $95 \% \mathrm{CI}$ & $p$-value & OR & $95 \% \mathrm{CI}$ & p-value \\
\hline Male gender & 4.383 & $4.231-4.541$ & $<0.001$ & 1.504 & $1.437-1.575$ & $<0.001$ \\
\hline \multicolumn{7}{|l|}{ Age } \\
\hline$<30$ & 1.000 & 1.000 & - & 1.000 & 1.000 & - \\
\hline $30-40$ & 1.800 & $1.701-1.901$ & $<0.001$ & 1.521 & $1.412-1.639$ & $<0.001$ \\
\hline $40-50$ & 2.319 & $2.196-2.451$ & $<0.001$ & 1.782 & $1.658-1.917$ & $<0.001$ \\
\hline $50-60$ & 2.937 & $2.772-3.113$ & $<0.001$ & 1.992 & $1.845-2.152$ & $<0.001$ \\
\hline$>60$ & 2.467 & $2.287-2.662$ & $<0.001$ & 1.652 & $1.499-1.821$ & $<0.001$ \\
\hline BMI & 1.436 & $1.427-1.445$ & $<0.001$ & 1.277 & $1.267-1.286$ & $<0.001$ \\
\hline Hypertension & 2.932 & $2.810-3.060$ & $<0.001$ & 1.387 & $1.313-1.465$ & $<0.001$ \\
\hline Central Obesity & 3.474 & 3.364-3.587 & $<0.001$ & 1.602 & $1.539-1.668$ & $<0.001$ \\
\hline Diabetes & 6.783 & $6.163-7.476$ & $<0.001$ & 1.706 & $1.645-1.769$ & $<0.001$ \\
\hline \multicolumn{7}{|l|}{ SUA } \\
\hline$<275$ & 1.000 & 1.000 & - & 1.000 & 1.000 & - \\
\hline $275-336$ & 6.039 & 5.393-6.788 & $<0.001$ & 2.616 & $2.300-2.986$ & $<0.001$ \\
\hline $336-402$ & 25.830 & $22.950-29.172$ & $<0.001$ & 4.444 & $3.866-5.123$ & $<0.001$ \\
\hline$>402$ & 59.308 & $38.574-94.116$ & $<0.001$ & 7.879 & $4.722-13.479$ & $<0.001$ \\
\hline High TC & 1.533 & $1.507-1.559$ & $<0.001$ & 1.081 & $1.031-1.135$ & 0.001 \\
\hline High TG & 6.543 & 6.325-6.769 & $<0.001$ & 2.649 & $2.538-2.765$ & $<0.001$ \\
\hline High LDL-C & 1.858 & $1.800-1.919$ & $<0.001$ & 1.332 & $1.271-1.396$ & $<0.001$ \\
\hline Low HDL-C & 1.672 & $1.612-1.735$ & $<0.001$ & 1.649 & $1.576-1.726$ & $<0.001$ \\
\hline Abnormal LF & 6.700 & $6.405-7.008$ & $<0.001$ & 3.228 & 3.053-3.414 & $<0.001$ \\
\hline
\end{tabular}

Note: "-" means no relevant data;OR: ratio ratio;Crude means only univariate regression analysis without adjustment of age, gender and other factors.Adjusted: The results with adjustion of gender, age, BMI, central obesity, diabetes, different levels of uric acid, high TC, high TG, highLDL-C, low HDL-C, and abnormal liver function.BMI:Body mass index;SUA:Serum uric acid;TC: Total Cholesterol;HDL: High density lipoproteins;LDL:Low density lipoproteins.LF: Liver function.

Table 3 The diagnostic accuracy of clinical indicators of NAFLD

\begin{tabular}{llll}
\hline Prediction & AUC & $95 \% \mathrm{CI}$ & $\mathrm{P}$ \\
\hline SUA & 0.749 & $0.745-0.752$ & $<0.01$ \\
HbA1C & 0.646 & $0.642-0.651$ & $<0.01$ \\
TC & 0.617 & $0.613-0.621$ & $<0.01$ \\
HDL & 0.727 & $0.723-0.731$ & $<0.01$ \\
LDL & 0.604 & $0.600-0.609$ & $<0.01$ \\
SBP & 0.688 & $0.684-0.692$ & $<0.01$ \\
\hline
\end{tabular}

Note: AUC, Area under the curve; CI, Confidence interval; SUA Serum uric acid; TC $₫$ Total Cholesterol; HDL, High-density lipoproteins; LDL, Low-density lipoproteins; HbA1c, Glycated hemoglobin; SBP, Systolic blood pressure.

\section{Figures}




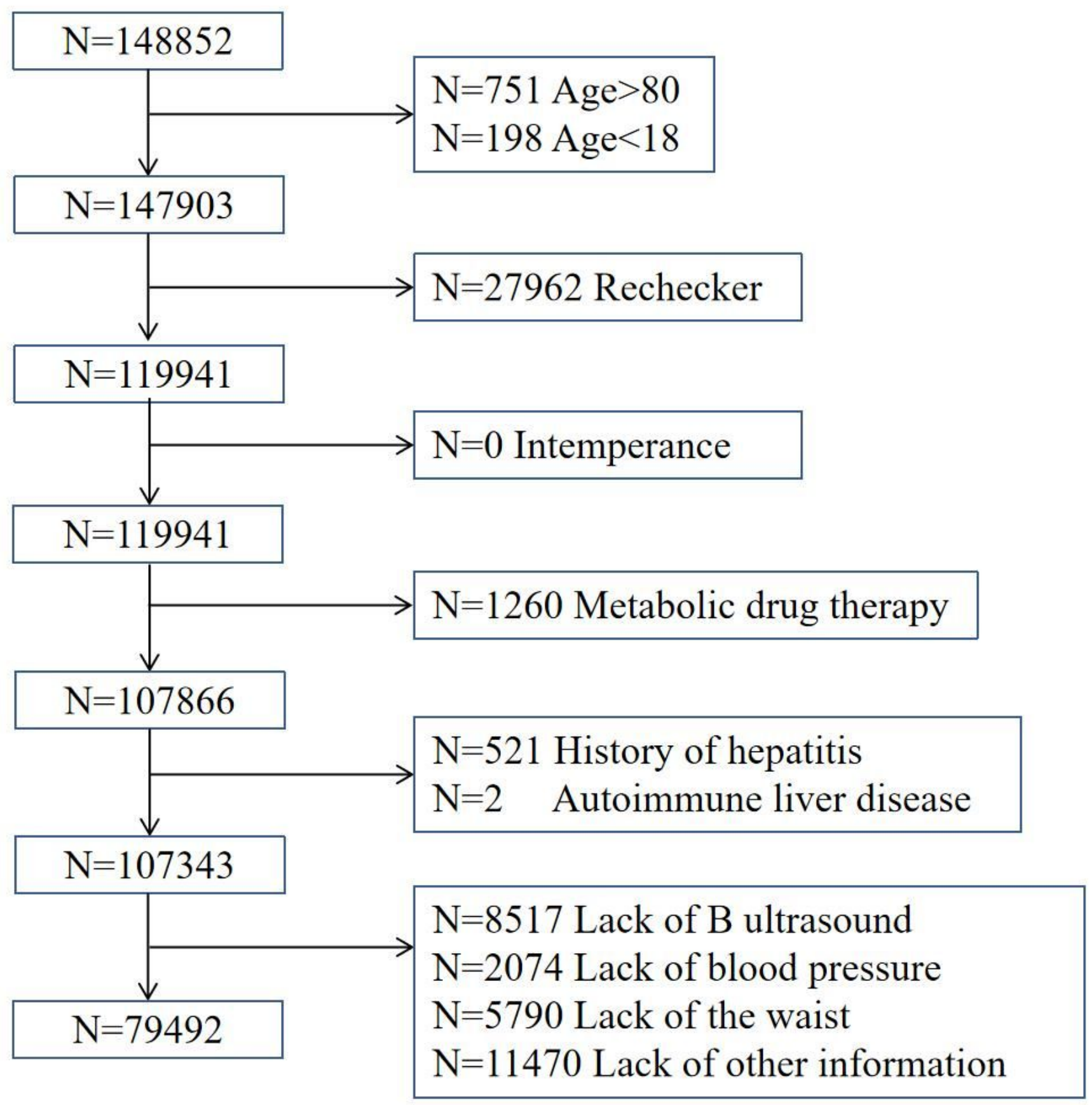

Figure 2

Screening flow chart of physical examination population 


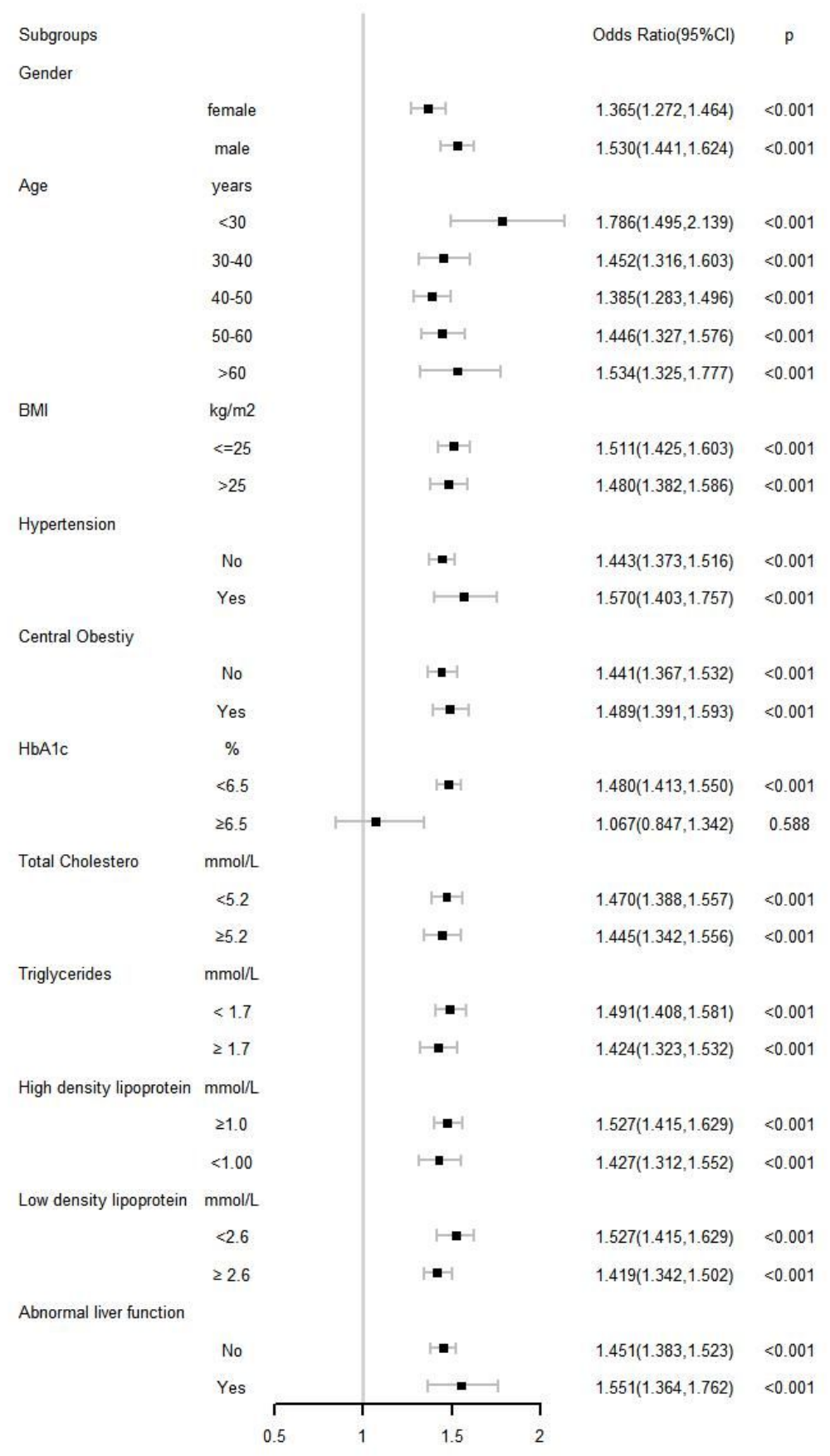

\section{Figure 4}

The ratio of high serum uric acid levels to NAFLD Note: Hypertesion refers to $S B P \geq 140 \mathrm{mmHg}$ or DBP $\geq 90 \mathrm{mmHg}$; Central Obesity refers to male waist circumference $>90 \mathrm{~cm}$ and female waist circumference $>80 \mathrm{~cm}$. 


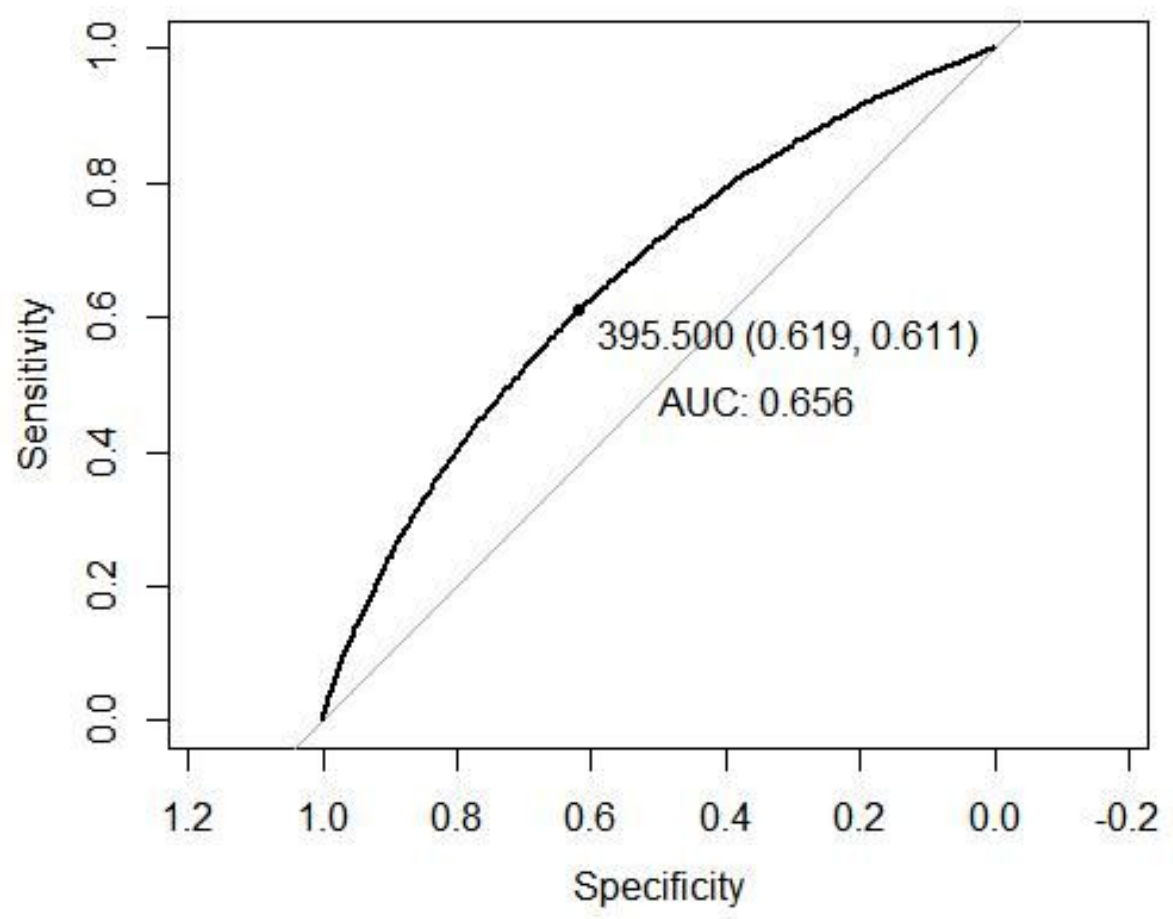

Figure 5

ROC curve of NAFLD diagnosed by UA level in male population

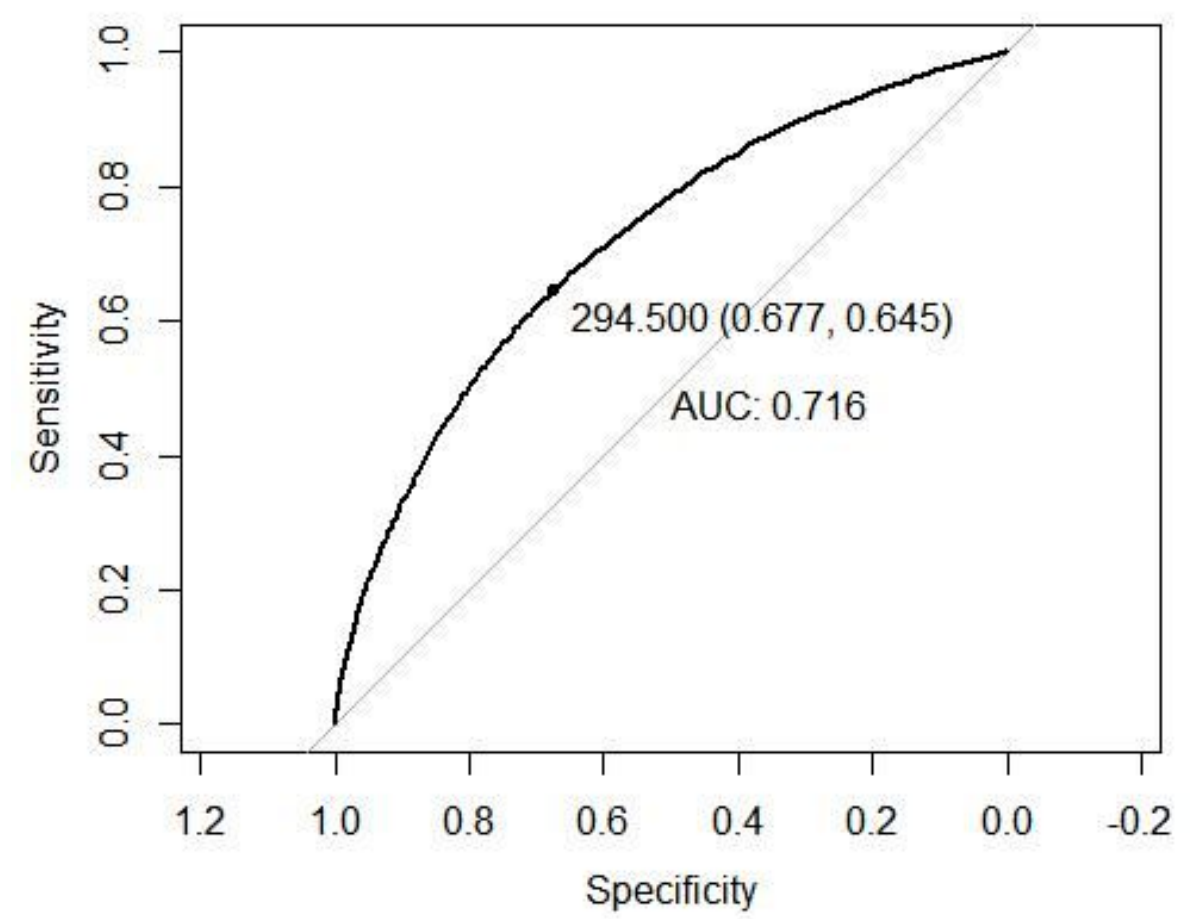

Figure 7

ROC curve of NAFLD diagnosed by UA level in female population 